\title{
Supplementary material: Heterogeneously integrated, superconducting silicon-photonic platform for measurement-device-independent quantum key distribution
}

\author{
Xiaodong Zheng ${ }^{\mathrm{a}, \dagger}$, Peiyu Zhang ${ }^{\mathrm{a}, \dagger}$, Renyou Ge ${ }^{\mathrm{b}, \dagger}$, Liangliang $\mathrm{Lu}^{\mathrm{a}, \dagger}$, Guanglong He ${ }^{\mathrm{a}, \dagger}$, Qi \\ Chen $^{\mathrm{a}, \dagger}$, Fangchao Qu ${ }^{\mathrm{a}}$, Labao Zhang ${ }^{\mathrm{a},{ }^{*}}$, Xinlun Cai ${ }^{\mathrm{b}, *}$, Yanqing $\mathrm{Lu}^{\mathrm{a}}$, Shining Zhu ${ }^{\mathrm{a}}$, Peiheng \\ $\mathbf{W u}^{\mathrm{a}}$, Xiao-Song $\mathrm{Ma}^{\mathrm{a}}$,* \\ ${ }^{a}$ Nanjing University, National Laboratory of Solid-state Microstructures, School of Physics, Research Institute of \\ Superconducting Electronics, School of Electronic Science and Engineering, College of Engineering and Applied \\ Sciences, Collaborative Innovation Center of Advanced Microstructures, Nanjing, China \\ ${ }^{\mathrm{b}}$ Sun Yat-sen University, State Key Laboratory of Optoelectronic Materials and Technologies, School of Electronics \\ and Information Technology, Guangzhou, China
}

\section{The heterogeneous superconducting-silicon-photonic chip}

In this section, we introduce the fabrication and characterization of the heterogeneous superconductingsilicon-photonic chip, including high-efficiency grating couplers, optical waveguides, multimode interference (MMI) couplers and waveguide-integrated superconducting nanowire single-photon detectors (SNSPDs).

The six fabrication steps of the high-efficiency grating couplers are shown in Fig. S1.

Fig. S2 shows the transmission spectrum of a reference device of $\sim 1500-\mu \mathrm{m}$-long waveguide with two grating couplers as the input and output, respectively. The total insertion loss of this reference device is about $-4.48 \mathrm{~dB}$ at a wavelength of $1536 \mathrm{~nm}$. If we neglect the relative small propagation loss of the on-chip waveguide, the insertion loss of each fiber-to-chip grating coupler is $-2.24 \mathrm{~dB}$ at $1536 \mathrm{~nm}$.

The fabrication steps of waveguide-integrated SNSPD are shown in Fig. S3 and explained in its caption. We use the setup in Fig. S4 to test the performance of the SNSPD, including its critical current, on-chip detection efficiency (OCDE) and dark count rate (DCR) in a closed-cycle fridge. We use a commercial electrical readout circuit to set bias current to detectors and obtain the response signal of detectors. Our sample is glued on the sample stage which is mounted on a 3-axis closed-loop low-temperature piezo nanopositioner. With the help of the nanopositioners, light can be coupled into the chip from fiber array. On the opposite side of fibre array, a RF probe is used to obtain the detectors' response signal.

After cooling the sample stage temperature down to $2.1 \mathrm{~K}$, we perform further low-temperature measurements, such as critical current, temporal response, OCDE and DCR. In Fig. S5, the I-V curves are measured by sweeping the voltage source between $-1 \mathrm{~V}$ and $1 \mathrm{~V}$. Each nanowire exhibits a superconducting state along the zero-voltage and abruptly transits to a normal-conducting state once the current is higher than critical current. It is clear to see that as the width of nanowire increases, the critical current increases.

As shown in Fig. S4, the OCDE of SNSPD is characterized by sending an attenuated continuous wave $(\mathrm{CW})$ laser and the DCR is measured when laser is turn-off. A fiber polarization controller (PC) is used to maximize the input power. First, we characterize the coupling efficiency of the reference grating coupler. After the photon flux is guided to fiber array in the cryostat, we 


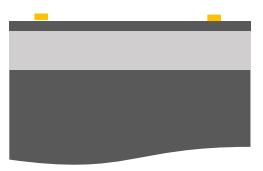

i

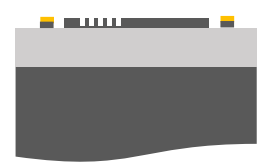

ii

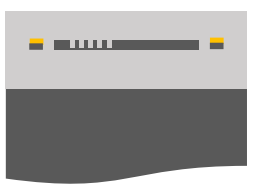

iii

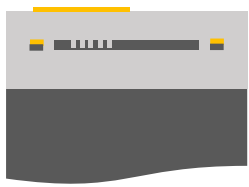

iv
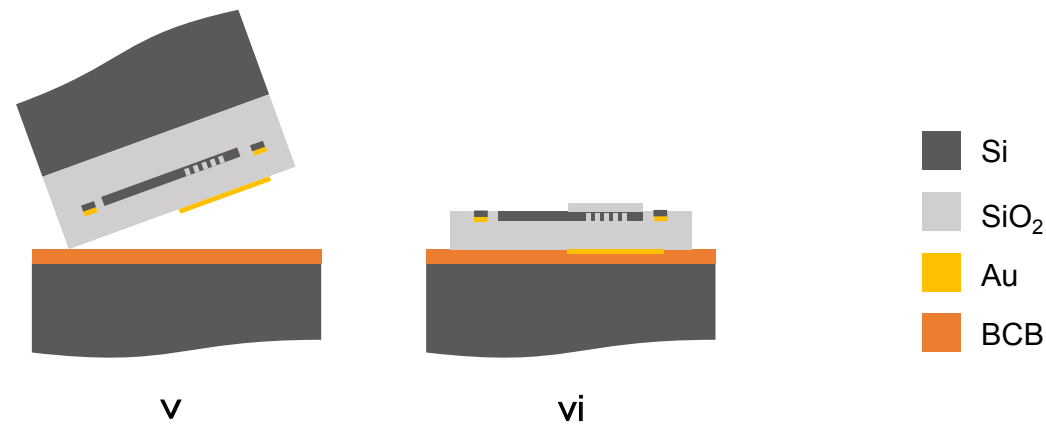

vi

Fig S1 Six-step fabrication process of the high-efficiency grating couplers. (i) The device is fabricated on a commercial silicon-on-insulator (SOI) wafer with $3 \mu \mathrm{m}$ thick buried oxide layer and $220 \mathrm{~nm}$ thick top silicon layer. We fabricate gold $\mathrm{(Au}$ ) pattern as the alignment markers for the subsequent steps. (ii) Silicon-photonic structures (including waveguides, MMIs and grating couplers) are patterned using electron beam lithography and etched using the inductively coupled plasma etching process. (iii) A thickness of $3 \mu \mathrm{m} \mathrm{SiO}{ }_{2}$ cladding is then deposited via the plasmaenhanced chemical vapor deposition and planarized via chemical mechanical polishing to an optimized thickness. (iv) Gold reflectors are then defined by lithography, metal evaporation and lift-off processes on the $\mathrm{SiO}_{2}$ cladding right above the grating couplers. (v) The chip is then flip-bonded to a silicon carrier wafer using benzocyclobuten (BCB). (vi) Finally, silicon substrate of the chip is removed by mechanical grinding and chemical etching, followed by dry etching the buried oxide layer.

measure the input power $P_{\text {in }}$ and output power $P_{\text {out }}$ with the attenuator is set at $0 \mathrm{~dB}$. The efficiency of the grating coupler can be calculated by the following formula

$$
\eta_{\text {in }} * \eta_{\text {out }}=\frac{P_{\text {out }}}{P_{\text {in }}}
$$

where $\eta_{\text {in }}$ and $\eta_{\text {out }}$ are the efficiency of the incidence grating coupler and emergent grating coupler, respectively. We neglect the on-chip propagation loss, so $\eta_{i n}=\eta_{\text {out }}=\eta$. As derived from Fig. S2, $\eta=-2.24 \mathrm{~dB}(1536 \mathrm{~nm})$. We also assume that the coupling efficiency of the main device's and the reference device's grating couplers are same. By adjusting the attenuator, we can find the photon number $N$ arriving at the detector in a similar way, with

$$
N=\frac{P_{i n}}{h \nu} * \eta * r
$$

where $r$ is the splitting ratio of MMI. We assume that the splitting ratio of multi-mode interference (MMI) is 50:50 in the following calculation. When the attenuation is $A$, with $N_{i n}=N * A$, the on-chip detection efficiency (OCDE) can be written as

$$
O C D E=\frac{C-C_{d}}{N_{\text {in }}}
$$




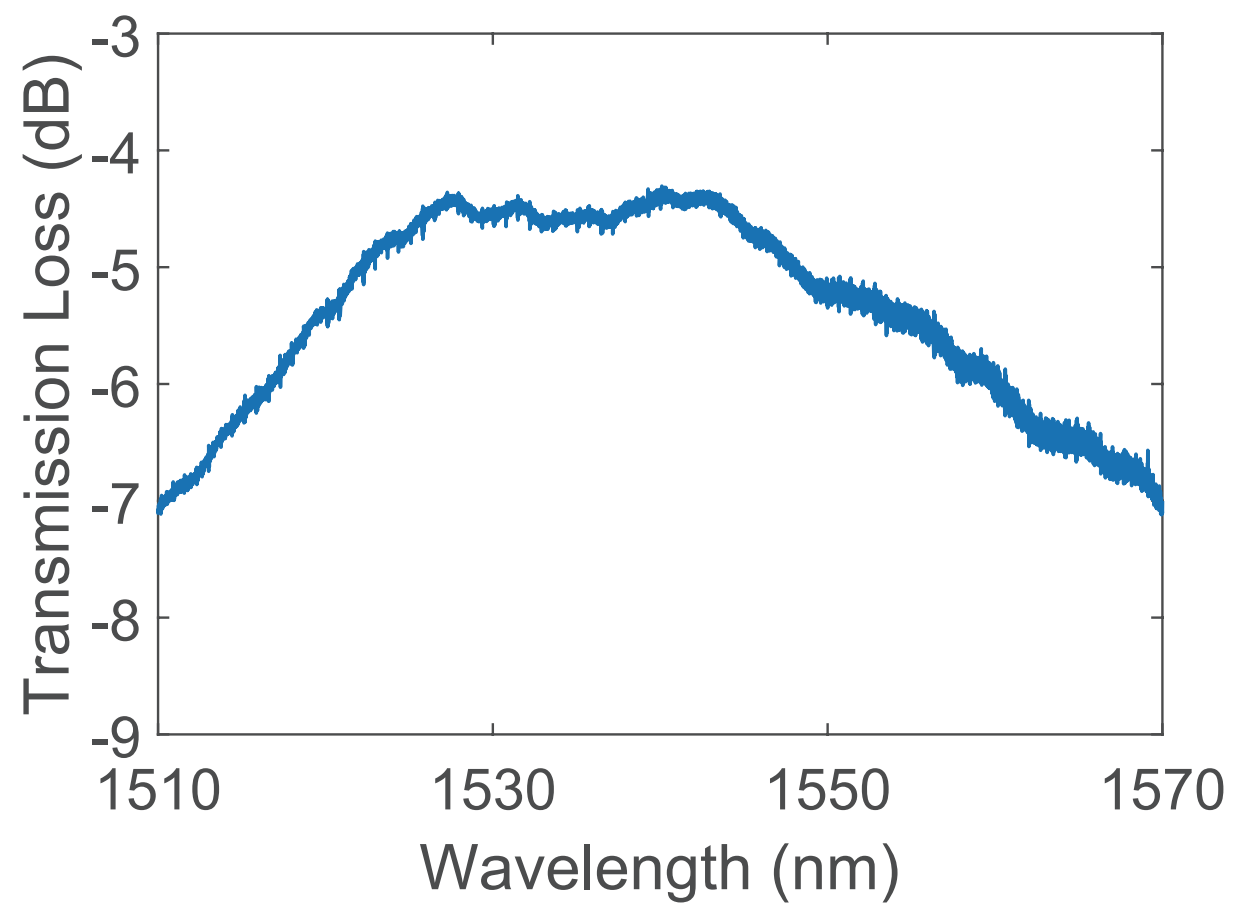

Fig S2 The transmission spectrum of the reference device measured in a cryogenic setup.

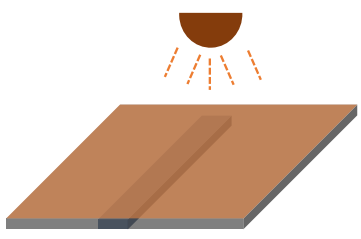

(i)

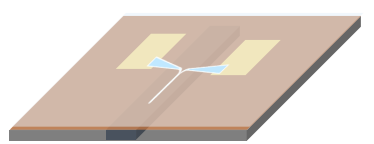

(iv)

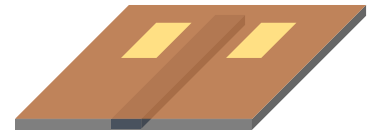

(ii)

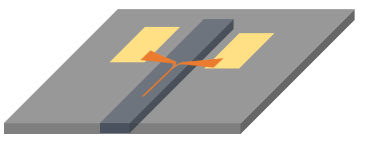

(v)

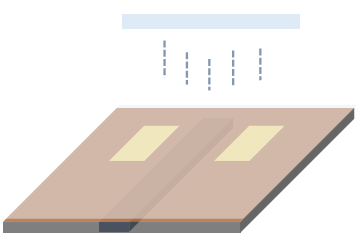

(iii)

Fig S3 Fabrication of NbN based SNSPD. (i) A nominal $6 \mathrm{~nm} \mathrm{NbN}$ film is deposited on the chip by DC reactive magnetron sputtering. Sheet resistance is $\mathrm{R}_{s}=341 \Omega / \square$, and critical temperature is about $6.1 \mathrm{~K}$. (ii) A series of fabrication process includes electron beam lithography (EBL), Titanium (Ti) and Gold (Au) deposition and lift-off, are used to fabricate gold pads for electronic contact. (iii) A $10 \mathrm{~nm} \mathrm{SiO}$ layer is deposited on $\mathrm{NbN}$ film by plasma-enhanced chemical vapor deposition to improve the adhesion between $2 \%$ hydrogen silsesquioxane (HSQ) and NbN. (iv) Then, the detector pattern is defined by EBL and developed using 2.38\% TMAH. (v) Lastly, $\mathrm{SiO}_{2}$ and the unwanted $\mathrm{NbN}$ are etched away in a timed reactive ion etching step with $C F_{4}$ plasma. 


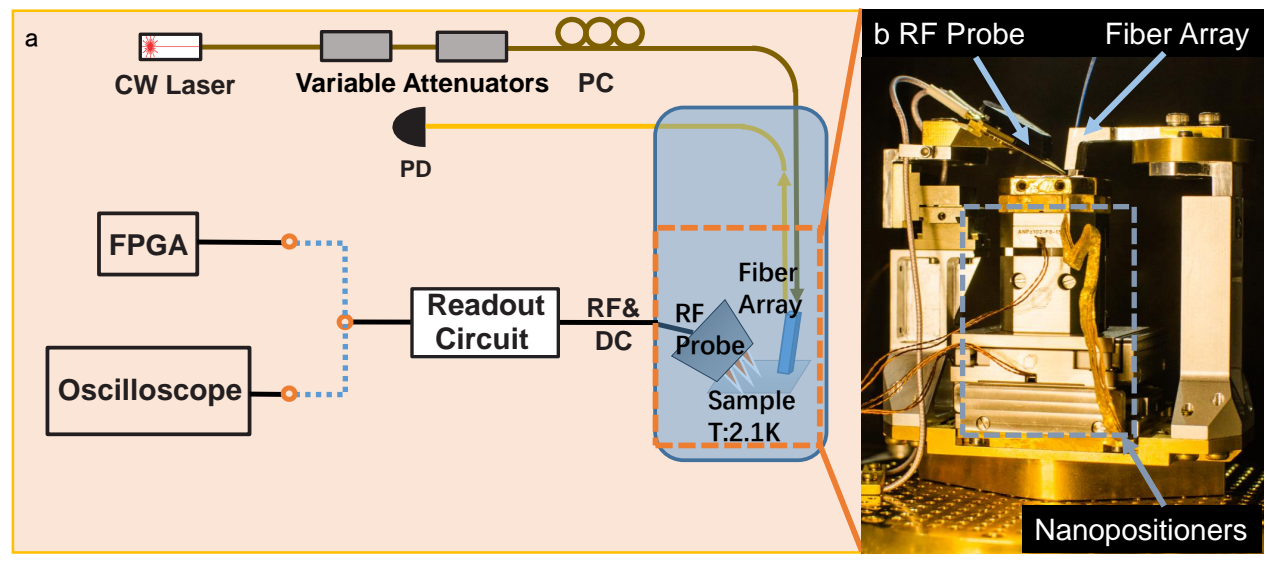

Fig S4 (a) Setup for critical current, OCDE and DCR measurements. (b) Photograph of sample stage which is mounted on a 3-axis closed-loop nano-positioner. See text for details.

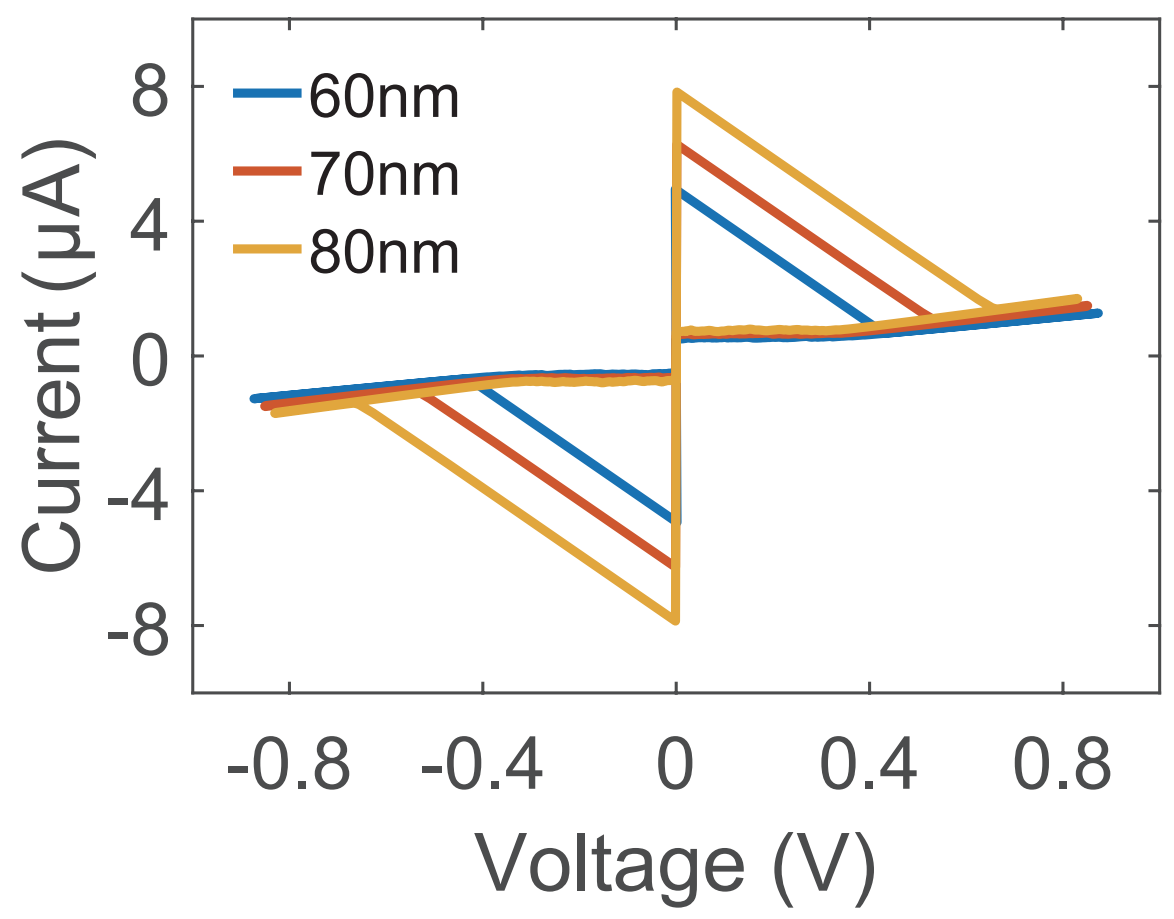

Fig S5 I-V curves of 3 nanowires with different widths. 
where $C$ is OCCR (on-chip count rate) and $C_{d}$ is DCR. We can get the value of $C$ and $C_{d}$ by using a timetagger (UQD). DCR is the count per second when we turn off the laser. We characterize the performance of our SNSPDs including OCDE and DCR with various bias currents at the wavelength of interests $(1536 \mathrm{~nm})$. The results are shown in Fig. S6. We change the bias current of our SNSPDs and obtain the OCDE and DCR as a function of normalized bias current. Saturation of count rate means that the internal quantum efficiency is close to $100 \%$. In our QKD experiment, SNSPD\#1 is biased at $7.1 \mu \mathrm{A}$, where OCDE is about 0.80 and the dark count rate is about $0.25 \mathrm{~Hz}$; SNSPD\#2 is biased at $6.8 \mu \mathrm{A}$, where OCDE is about 0.81 and the dark count rate is about $0.24 \mathrm{~Hz}$.

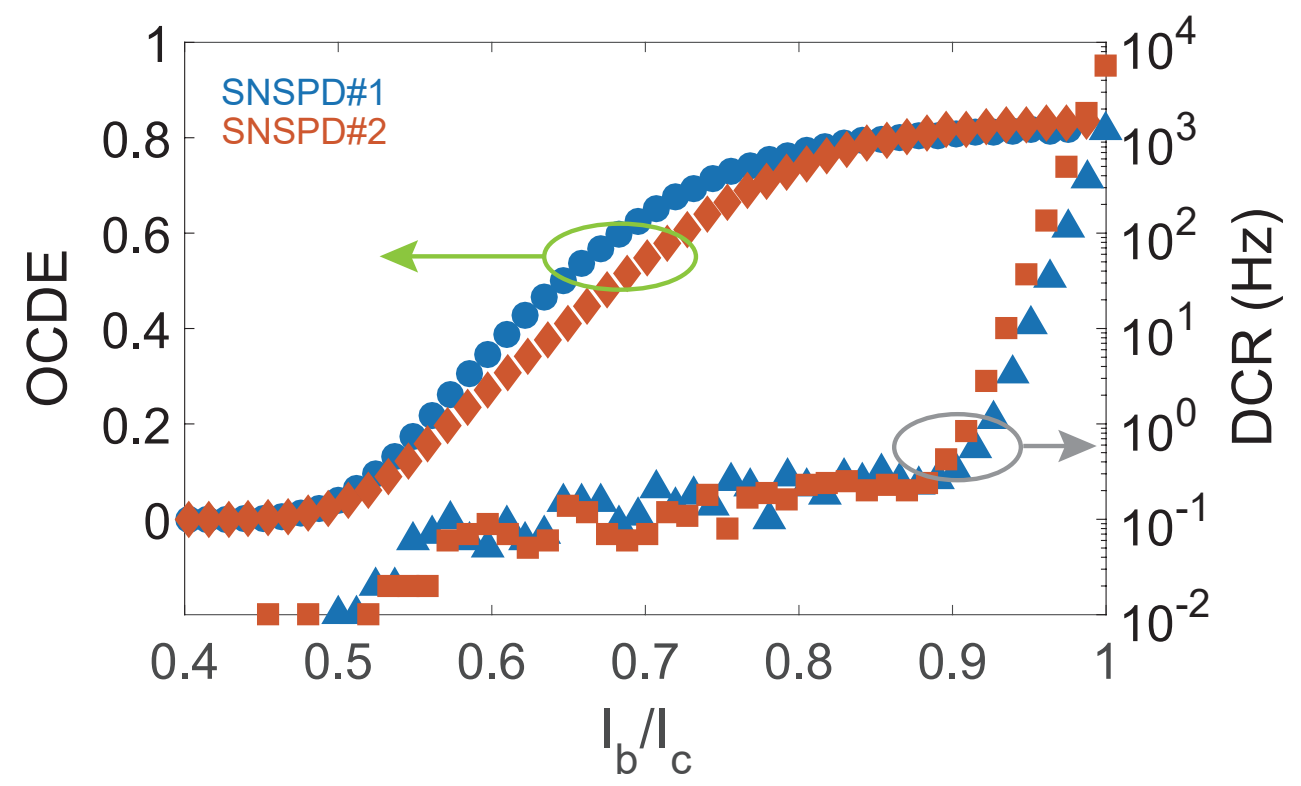

Fig S6 The OCDE and the DCR as a function of $I_{b} / I_{c}$ for the SNSPDs. $I_{c}$ is the critical current of SNSPDs. The data of OCDE and DCR are the averages of 100 one-second measurements.

\section{Off-chip optical setup}

In our experiment, the light sources are two CW lasers with a nominal linewidth of $100 \mathrm{~Hz}$ centered at $1536.47 \mathrm{~nm}$. For our experiment, it is important to keep the frequencies of both Alice's and Bob's lasers to be the same. We keep track on the frequency difference between these two lasers by beat measurement and employ a feedback system to regulate Bob's laser's frequency. The maximum frequency difference is less than $10 \mathrm{MHz}$ over about 15 hours with the help of the feedback system, as shown in Fig. S7.

For each encoder module, we use one intensity modulator to chop out the required short pulses with about 370 ps full-width at half maximum (FWHM). Then we use another intensity modulators to modulate the mean photon number per pulse for decoy states $(\mu$ and $\nu)$ and vacuum state $(o)$. In Fig. S8, we show the results of weak coherent pulses measured by our SNSPDs, in which a $>20$ $\mathrm{dB}$ extinction ratio has been obtained. 


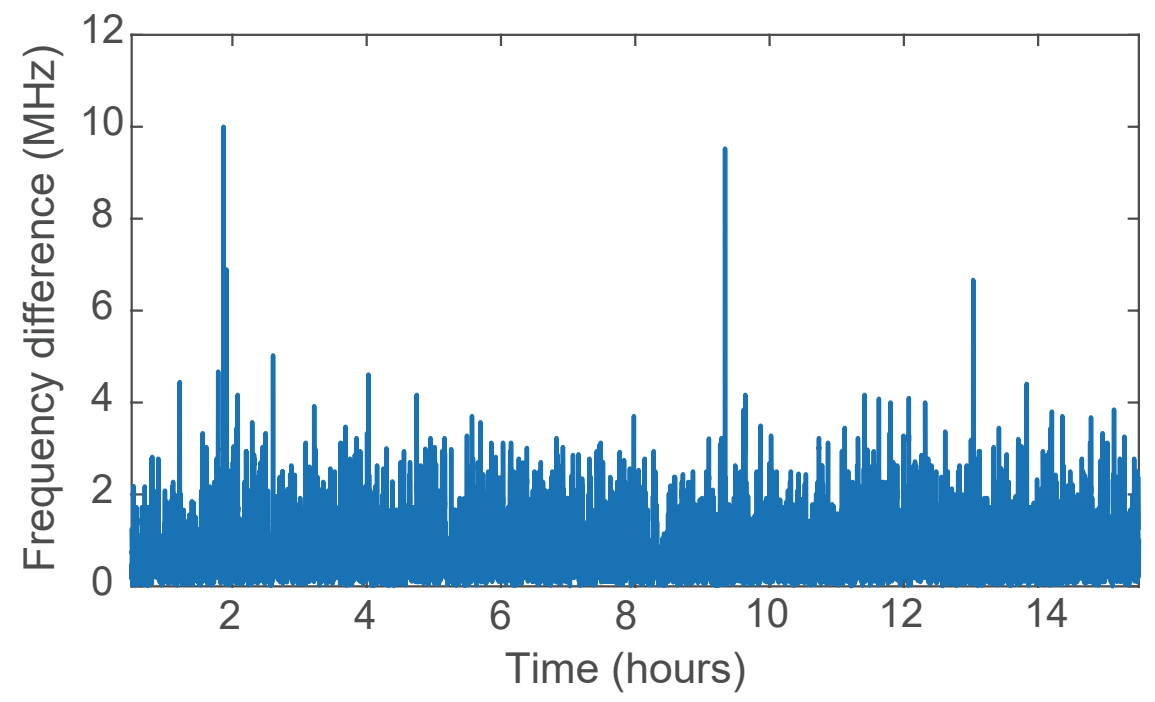

Fig S7 Frequency difference between the lasers of Alice and Bob with the feedback control. A maximum frequency drift no more than $10 \mathrm{MHz}$ is observed in $\sim 15$-hour monitoring data.

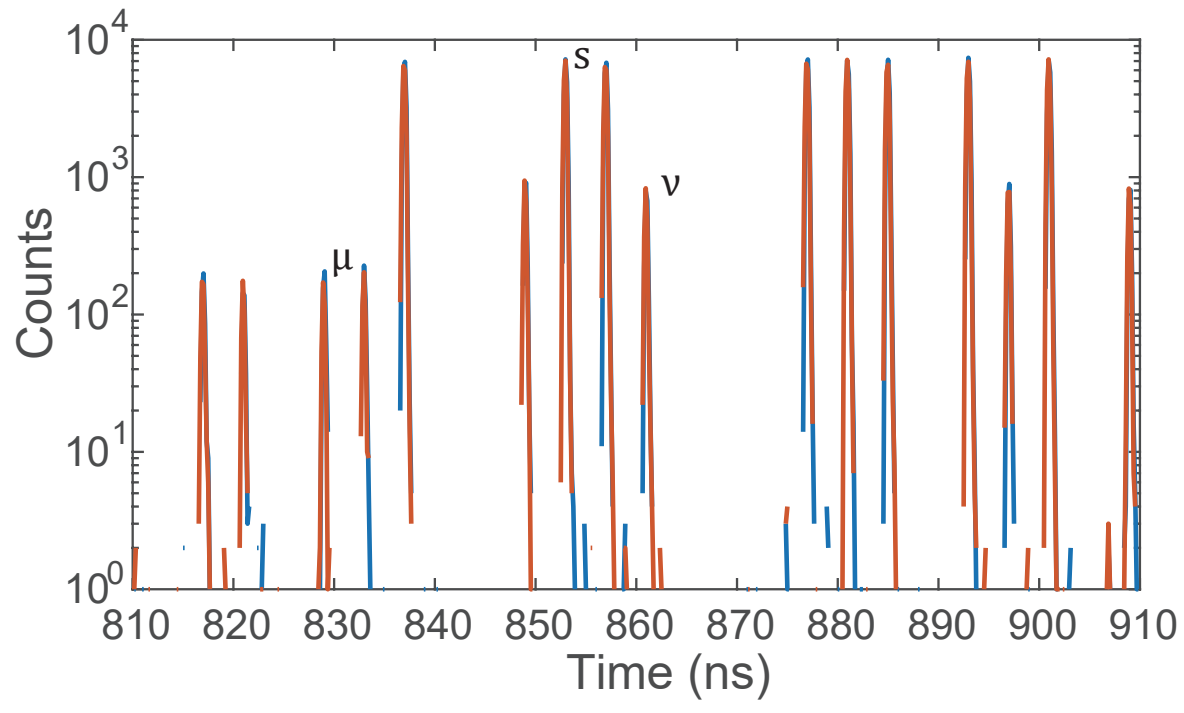

Fig S8 Histogram of the weak coherent pulse train (including signal state $s$, decoy state $\mu$ and $\nu$.) with a rate of 125 $\mathrm{MHz}$ is detected by the waveguide-integrated SNSPDs. The extinction ratio of the pulses is more than $20 \mathrm{~dB}$. Red line and blue line are histogram between reference signal and two SNSPDs respectively. The detector electrical signals are collected by a timetagger with a temporal resolution of $156 \mathrm{ps}$. Count integration time is $200 \mathrm{~s}$. 


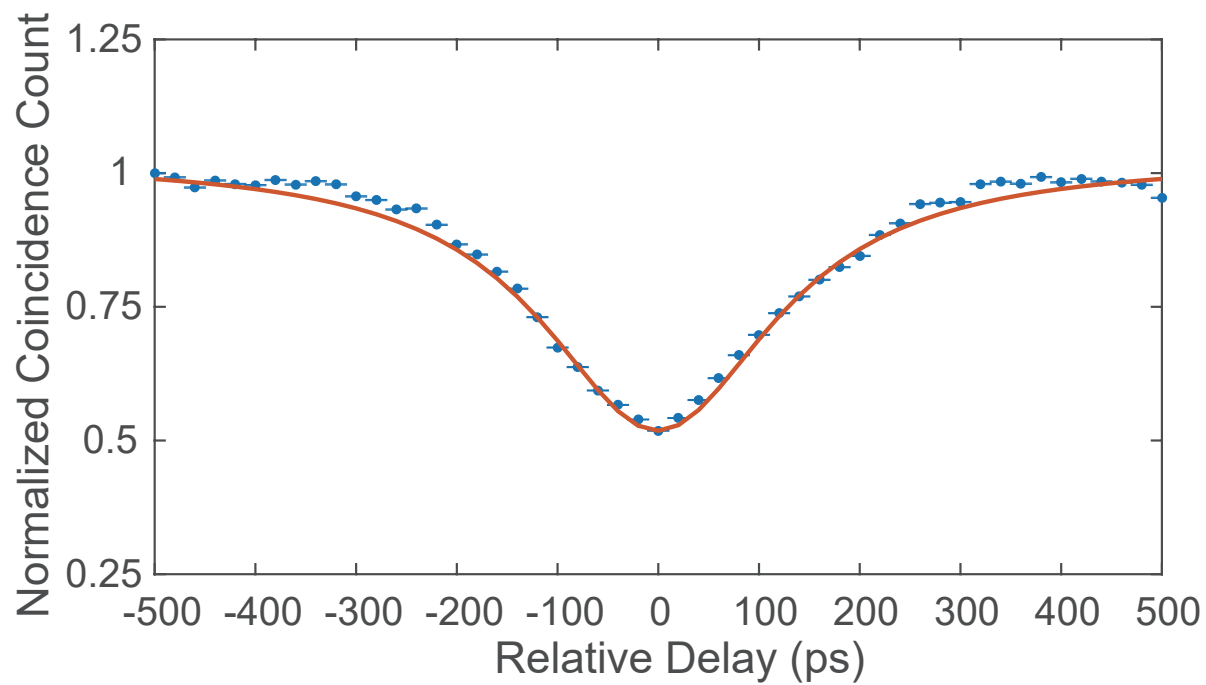

Fig S9 Hong-Ou-Mandel (HOM) interference as a function of relative electronic delays between Alice and Bob. A HOM interference visibility of $V=48.1 \% \pm 0.5 \%$ is observed. The red curve is a fit.

\section{Four intensity decoy-state analysis}

We use the four-intensity decoy-state protocol $^{46}$ and consider the symmetric case where Alice and Bob have equal channel transmissions to Charlie. Experimentally, Alice and Bob each use one signal intensity $s$ in $Z$ basis for key generation and three decoy intensities $(\mu, \nu, o)$ in $X$ basis for error test, where $o$ represents vacuum. In practical implementation, the key size is finite and the statistical fluctuation should be considered differently if pulse pairs from Alice $(l)$ and Bob $(r)$ in different intensity combinations $l r$, with $l, r \in\{s, \mu, \nu, o\}$. The key idea of the finite size analysis is to estimate a lower bound for the yield of single-photon pairs $\left(\left[y_{11}\right]_{\min }\right)$ and an upper bound for the phase error rate of single-photon pairs $\left(\left[e_{11}^{p h}\right]_{\max }\right.$ ) from gain of the pulse pairs in the $X$ basis by applying the Chernoff bound to perform parameter estimation ${ }^{47,48}$. According to Ref.46, by jointly considering the observed data in $l r \in D=\{o o, o \mu, \mu o, o \nu, \nu o, \mu \mu, \nu \nu\}$, one can acquire tighter bound from the joint constrains than independent constrains, and hence produce a higher key rate. The final key rate can be extracted by

$$
R=p_{s} p_{s}\left\{s^{2} e^{-2 s}\left[y_{11}\right]_{\min }\left[1-h\left(\left[e_{11}^{p h}\right]_{\max }\right)\right]-f y_{s s} h\left(E_{s s}\right)\right\},
$$

where $p_{s} p_{s}$ is the probability of both Alice and Bob sending signal states. $h(\cdot)$ is the Shannon binary entropy function, and $f=1.16$ is the efficiency of error correction. $y_{s s}$ and $E_{s s}$ denote the gain and QBER for ss source, which can be directly obtained in experiments.

\section{Experimental results}

As shown in Fig. S9, we obtain a HOM interference visibility of V=48.1\% $\pm 0.5 \%$ with 4 ns time-bin separation at a rate of $250 \mathrm{MHz}$, indicating the high quality of our integrated chip for MDI-QKD. The blue dots are the experimental data and the red curve is a fit.

We employ our system to implement a complete MDI-QKD system including four intensity decoy states and phase randomization.In Table S1, we show the mean photon number and proportion of different states and the sent total pulse pairs number at different loss. In Table S2, we show 
detailed experimental results. The system has a $125 \mathrm{MHz}$ repetition rate. The system is run for 6.68 hours at total attenuations of $24.0 \mathrm{~dB}$ (including chip insertion loss $\sim 4.5 \mathrm{~dB}$, the actual transmission loss corresponds to $98 \mathrm{~km}$ standard fibre), and a total of $3 \times 10^{12}$ pulse pairs are sent from each sender. The key rate is 6166 bps. The system is also run for 6.68 hours at total attenuations of $35.0 \mathrm{~dB}$ (including chip insertion loss $\sim 4.5 \mathrm{~dB}$, the actual transmission loss corresponds to 153 $\mathrm{km}$ standard fibre), and a same total of $3 \times 10^{12}$ pulse pairs are sent from each sender. The key rate is $170 \mathrm{bps}$. As for higher attenuation, $44.0 \mathrm{~dB}$ (including chip insertion loss $\sim 4.5 \mathrm{~dB}$, the actual transmission loss corresponds to $198 \mathrm{~km}$ standard fibre), the run time is increased by one order (66.8 hours). So the sent pulse pairs number becomes $3 \times 10^{13}$. The key rate is $34 \mathrm{bps}$. In Table $\mathrm{S} 3$, We show the comparison of state-of-the-art MDI-QKD experiments. We emphasize that our secure key rates with $125 \mathrm{MHz}$ clocked system are very close to the best MDI-QKD experiments with $\mathrm{GHz}$ clock rate ${ }^{17,31,56}$. In Fig. S10, we set the clock rate equal to $125 \mathrm{MHz}$ for all the simulated rates. To draw these curves, we have set transmissivity $\eta=10^{-\operatorname{Loss}(d B) / 10}$. The PLOB curve and the decoy-state MDI-QKD curve ${ }^{57}$ have been obtained under ideal conditions, i.e., with zero detector and channel noise, maximum detection efficiency (detection efficiency is 1) and unitary error correction efficiency $(f=1)$. PLOB bond: $R_{P}=-\log _{2}(1-\eta)$. Decoy-state MDI-QKD: $R_{D}=\eta /\left(2 e^{2}\right)$.

Table S1 List of mean photon number and proportion of signal state and decoy state

\begin{tabular}{|l|c|c|c|}
\hline Loss $^{*}(\mathrm{~dB})$ & 24.0 & 35.0 & 44.0 \\
\hline$s$ & 0.714 & 0.66 & 0.624 \\
\hline$\mu$ & 0.034 & 0.048 & 0.054 \\
\hline$\nu$ & 0.172 & 0.196 & 0.208 \\
\hline$P_{s}$ & 0.828 & 0.774 & 0.736 \\
\hline$P_{\mu}$ & 0.14 & 0.176 & 0.204 \\
\hline$P_{\nu}$ & 0.014 & 0.03 & 0.039 \\
\hline$N$ & $3 \times 10^{12}$ & $3 \times 10^{12}$ & $3 \times 10^{13}$ \\
\hline
\end{tabular}


Table S2 List of the total gains and error gains of Bell states. The notion $N_{i j}$ denotes the number of pulse pairs sent out from $i j$ intensity combination.

\begin{tabular}{|l|c|c|c|}
\hline Loss $(\mathrm{dB})$ & 24.0 & 35.0 & 44.0 \\
\hline$N_{s s} y_{s s}$ & 1340443872 & 81820241 & 83430549 \\
\hline$N_{s s} y_{s s} E_{s s}$ & 89872 & 8187 & 29458 \\
\hline$N_{\mu \mu} y_{\mu \mu}$ & 189673 & 47419 & 87324 \\
\hline$N_{\mu \mu} y_{\mu \mu} E_{\mu \mu}$ & 54435 & 13193 & 24656 \\
\hline$N_{\nu \nu} y_{\nu \nu}$ & 33781 & 25250 & 37087 \\
\hline$N_{\mu o} y_{\mu o}+N_{o \mu} y_{o \mu}$ & 11123 & 2673 & 4632 \\
\hline$N_{\nu o} y_{\nu o}+N_{o \nu} y_{o \nu}$ & 17115 & 6481 & 9494 \\
\hline$N_{o o} y_{o o}$ & 0 & 0 & 0 \\
\hline$y_{11}$ & $1.60 \times 10^{-3}$ & $9.29 \times 10^{-5}$ & $1.28 \times 10^{-5}$ \\
\hline$e_{11}^{p h}$ & 0.1616 & 0.2321 & 0.1557 \\
\hline Key rate/ pulse & $4.93 \times 10^{-5}$ & $1.36 \times 10^{-6}$ & $2.72 \times 10^{-7}$ \\
\hline
\end{tabular}

${ }^{*}$ Including chip insertion loss $\sim 4.5 \mathrm{~dB}$

Table S3 Comparison of state-of-the-art MDI-QKD experiments.

\begin{tabular}{lcccc}
\hline Reference & Clock rate $(\mathrm{MHz})$ & Loss $(\mathrm{dB})$ & Key rate $(\mathrm{bps})$ & Key rate/ pulse \\
\hline${\text { Comandar } \text { et } \text { al. }^{17}}^{17}$ & 1000 & 20.4 & $4567^{\mathrm{a}}$ & $4.57 \times 10^{-6}$ \\
\hline Wei et al. $^{31}$ & 1250 & 20.4 & $6172^{\mathrm{b}}$ & $4.94 \times 10^{-6}$ \\
& & 28.0 & 268 & $2.14 \times 10^{-7}$ \\
& & 36.0 & 31 & $2.48 \times 10^{-8}$ \\
\hline${\text { Woodward } \text { et } \text { al. }^{56}}^{5}$ & 1000 & 30.0 & 1971 & $1.97 \times 10^{-6}$ \\
& & 40.0 & 58 & $5.80 \times 10^{-8}$ \\
\hline This work & 125 & $24.0^{\mathrm{c}}$ & 6166 & $4.93 \times 10^{-5}$ \\
& & $35.0^{\mathrm{c}}$ & 170 & $1.36 \times 10^{-6}$ \\
& & $44.0^{\mathrm{c}}$ & 34 & $2.72 \times 10^{-7}$ \\
\hline
\end{tabular}

a No random modulations.

b Simulation.

${ }^{\mathrm{c}}$ Including chip insertion loss $\sim 4.5 \mathrm{~dB}$. 

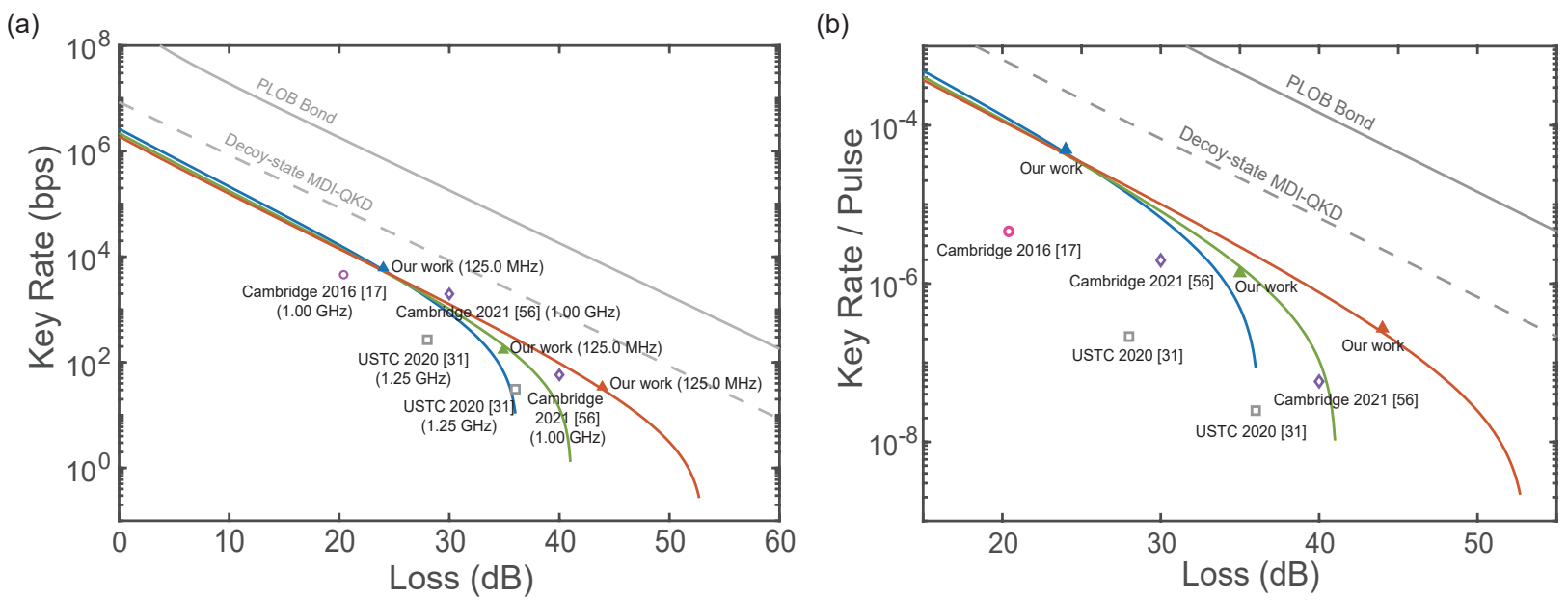

Fig S10 Our experiment results and comparison of state-of-the-art MDI-QKD experiments. (a) Key rate versus loss. (b) Key rate per pulse versus loss. 